\title{
On comparing the real and probed packet drop rates of a bottleneck router: the TCP traffic case
}

\author{
S.Y. Wang \\ Department of Computer Science and Information Engineering, National Chiao Tung University, 1001 Ta Hsueh Road, Hsinchu 30050, Taiwan, ROC
}

Received 19 January 2002; revised 6 June 2002; accepted 24 June 2002

\begin{abstract}
In this paper, we address the question of whether the packet drop rate of a bottleneck router measured (experienced) by probe packets can accurately reflect the bottleneck router's real packet drop rate. To answer this question, we built a testbed network and conducted several real experiments on it. In these experiments, using various traffic types and loads, we compared the probed and the real packet drop rates of the bottleneck router. We also collected the internal queue length variation process of the bottleneck router and used it to do several post analyses to answer some 'what-if' questions. This paper presents some interesting findings derived from this study.
\end{abstract}

(C) 2002 Elsevier Science B.V. All rights reserved.

Keywords: Network measurement; Traffic measurement; Performance evaluation

\section{Introduction}

Injecting probe packets to a network is a method to measure the quality of a network. In this method, the packet drop rates and delays experienced by probe packets are used as estimates of the real packet drop rate and delay of the network. Because this method does not need any support from the network, network customers can easily use this method to estimate the quality of their networks. To help customers choose an Internet service provider (ISP) with the best quality, some third parties also use the probe method to routinely measure and publish the quality of some ISPs' networks.

Although the probe method is widely used, it has not been adequately studied in the literature and many important questions still remain unsolved. For example, suppose that we have an infinite number of probe packets, will the measured result converge and accurately reflect the real packet drop rate? How many probe packets are needed to obtain a converged result? Does the distribution of the inter-probe packet times (i.e. different sampling techniques) affect the measured results and the convergence speed? Not knowing these answers, a customer who uses a probe method to estimate the quality of his network may obtain wrong measured results, interpret the measured results

E-mail address: shieyuan@csie.nctu.edu.tw (S.Y. Wang). wrongly, or waste time and effort on collecting unnecessary samples.

To answer these important questions, we built a testbed network to collect the detailed packet arrival, departure, and dropping events, and queue length variation process of a bottleneck router. With these information (which cannot be obtained by an end-to-end probe method), we conducted several on-line probe experiments to study the difference between the measured and real packet drop rates of a bottleneck router, and perform several off-line post analyses to answer some 'what if...' questions.

This paper presents some interesting findings about packet drop rate measurements. These findings are derived from the results collected on three different routers and switches that were tested in our experiments (a FreeBSD router, a CISCO AGS + router, and a 3COM 3300 SM switch). In these experiments, we used two different kinds of network traffic to generate congestion on the bottleneck router/switch. These two kinds of traffic are 'greedy TCP traffic' and 'greedy HTTP traffic', respectively. The greedy TCP traffic is used to simulate the traffic that would be generated by FTP programs that transfer huge files. The greedy HTTP traffic is used to simulate the traffic that would be generated by Web clients that continuously download small-sized web pages.

This paper is organized as follows. Section 2 surveys related work. Section 3 defines the terms that are frequently 
used in the paper. Section 4 describes the experimental setup of our testbed networks. Section 5 presents our measurement method and tool. Section 6 shows our experimental results and derived findings when the network traffic is greedy TCP traffic. Section 7 shows our experimental results and derived findings when the network traffic is greedy HTTP traffic. Finally, Section 8 concludes this paper.

\section{Related work}

There have been some researches on studying the packet dropping behavior in a network. In Refs. [1-4], the authors studied the packet dropping behavior of the Internet by injecting unicast probe packets into the Internet and observing their dropping behavior. In Ref. [5], the authors inferred the packet drop rate of links by injecting multicast probe packets onto a multicast tree. In Ref. [6], the authors used analytical methods (queueing theory) to analyze the packet loss process in a single server queueing system under the assumption that the packet arrival is a Poisson process. In Ref. [7], the authors used a collected packet trace to study the relative performance of various sampling techniques. In Ref. [8], the author studied the loss dependence of packets that are transmitted in the Internet. Our work differs from these work in that we study the difference between the probed packet drop rate and the real packet drop rate of a bottleneck router; however, these work do not investigate this problem.

\section{Terminology}

Several terms that are frequently used in this paper are defined in this section.

Real packet drop rate is the packet drop rate of a bottleneck output port. Suppose that, over a long period of time, $N$ packets arrive at a bottleneck output port and $D$ of them are dropped before they are transmitted, then the packet drop rate of this bottleneck output port is $D / N$. In some contexts, when there is no ambiguity, we simply call it 'packet drop rate'. Depending on contexts, it may be used to represent the packet drop rate of a bottleneck output port, a bottleneck router, or a network.

Measured packet drop rate is the packet drop rate of a bottleneck output port measured (experienced) by probe packets over a period of time. Suppose that $N$ probe packets traverse through a bottleneck output port and $D$ of them are dropped, then the packet drop rate of the bottleneck output port measured (experienced) by the probe packet stream is $D / N$. In some contexts, we use it to represent the measured packet drop rate of a bottleneck output port, the bottleneck router, or the network.

Queue-full percentage is the percentage of time in which the bottleneck output port's queue is full. This information can be calculated from the queue length variation process of the output queue. A queue length variation process has many queue-full periods. A queue-full period starts when the current queue length goes up to maxqlen and ends when the current queue length goes down to maxqlen-1, where maxqlen is the configured maximum queue length for the output queue. Any packet arriving in this period will be dropped due to a full queue. Suppose that, during an experiment, the total time of the experiment is $T$ seconds and the total time of its queue-full periods is $F$ seconds, then the queue-full percentage is $F / T$.

FIFO scheme is the most commonly used first-in-firstout packet scheduling and drop-tail packet dropping scheme. To save space, when there is no ambiguity, we simply use 'the FIFO scheme' to represent the FIFO and drop-tail scheme.

Greedy TCP traffic is the kind of traffic that is generated by many greedy TCP flows. A greedy TCP flow is a TCP connection that has infinite data to send and uses as much bandwidth as allowed by its TCP congestion control.

Greedy HTTP traffic is the kind of traffic generated by many greedy HTTP web clients. A greedy HTTP web client is a program that continuously issues a new web request to download a web page immediately when the current request is completed. Each web request will cause a TCP connection to be set up before the requested web page can be downloaded. In this study, the size of the web pages is a fixed value of $16 \mathrm{~KB}$. The web server program used is Apache 1.3. The web client program used is a script program that continuously issues the ' $\mathrm{ftp}$ http://host[:port]/ file' command. This ftp command will use the HTTP protocol to download the requested file from a web server. More information can be found in Ref. [9].

\section{Experimental testbeds}

In our experiments, we used two different kinds of traffic to study the problem. The first kind is greedy TCP traffic and the second kind is greedy HTTP traffic. (A HTTP session's transfer amount is small but it also uses TCP as its transport protocol.) We chose TCP traffic to study because in today's Internet, TCP traffic is the most dominant traffic.

\subsection{Greedy TCP traffic}

We studied this kind of traffic because it is similar to the traffic that would be generated when many people use ftp to transfer huge files.

Two single-bottleneck configurations are used in this suite of experiments. They are shown in Fig. 1. In both configurations, there are seven sending hosts on the left and one receiving host on the right. Between each sending host and the receiving host, there are $N$ greedy TCP flows and therefore there are $7 * N$ greedy TCP flows in total passing through the bottleneck router/switch, where $N$ is varied from 4, 8 to 12. All of these hosts are PC running FreeBSD 4.2 
Configuration (a)

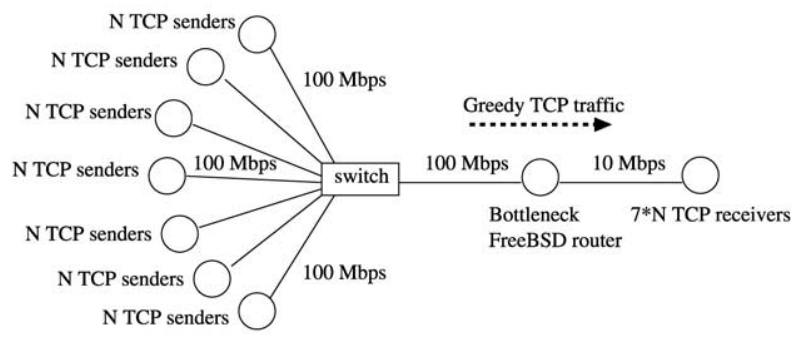

Configuration (b)

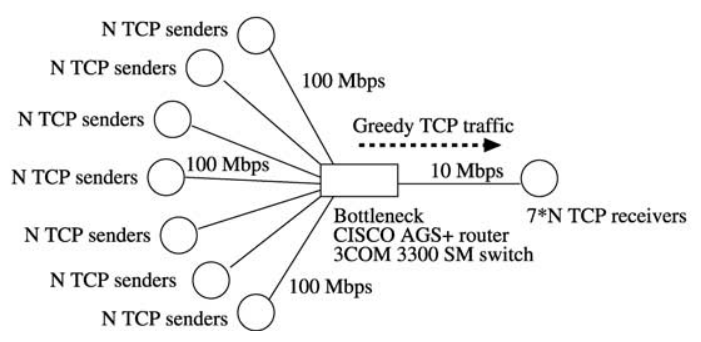

Fig. 1. The two configurations used in the greedy TCP traffic experiments

and use Intel EtherExpress 100/10 Mbps Ethernet cards. All links are 10-m full-duplex Ethernet cables and thus their propagation delays are negligible. This experimental configuration is chosen to create congestion conditions and the bottleneck router in this configuration is expected to represent a typical bottleneck router operating in a real network.

Configuration (a) and (b) differ in what is used for the bottleneck router/switch. In (a), because we want to collect the detailed packet arrival, departure, and dropping processes of the bottleneck router but commercial routers and switches do not provide this information, we used a 1.6 GHz PC running FreeBSD 4.2 as the bottleneck router. Because a PC does not have enough PCI/ISA slots to plug-in eight Ethernet cards to connect to the seven sending hosts and one receiving host, we set the bandwidth of the link between the FreeBSD router and the receiving host to be only $10 \mathrm{Mbps}$, and let the seven sending hosts and the FreeBSD router connect to the switch (Intel 8-port InBusiness switch) all at $100 \mathrm{Mbps}$ speed.

Under this network and traffic configurations, the bandwidth of the most-congested link (i.e. the link between the FreeBSD router and the receiving host) is purposely set to only $10 \mathrm{Mbps}$. Because TCP implements congestion control, the aggregate throughput of these competing TCP flows cannot exceed $10 \mathrm{Mbps}$ in the long term. In the short term, although the instantaneous aggregate throughput may have a chance to exceed $10 \mathrm{Mbps}$, the difference should be minimal. Because the bandwidth of the switch's bottleneck output port is $100 \mathrm{Mbps}$, which is 10 times of the bandwidth of the most-congested link, packets of these competing TCP flows will not have a chance to be dropped in the switch. The reasons are that before they have a chance to be dropped in the switch, they should have already been dropped on the most-congested link.

In configuration (b), we used a CISCO AGS + router and a 3COM $3300 \mathrm{SM}$ switch to repeat the experiments conducted on configuration (a). We did this to see whether the findings obtained from a FreeBSD router also apply to some commercial routers/switches. The CISCO AGS + router was manufactured in Spring 1995 and stopped servicing our campus in Spring 1999. We disabled the 3 COM switch's IEEE $802.3 \times$ flow control so that there is no link-level flow control in our experiments. Unlike what we did in configuration (a), since a commercial router/switch has many ports, we directly connected the seven sending hosts and one receiving host to the router/switch without using an extra switch. Since these commercial routers/switches do not provide the detailed packet arrival, departure, and dropping process information, we focused our study only on the packet drop rate of the router/switch. This information can be calculated by the method presented in Section 5.2 if it is not provided by the router/switch.

\subsection{Greedy HTTP traffic}

We studied this kind of traffic because it is similar to the traffic that would be generated when many people continuously download small-sized web pages.

In this suite of experiments, we used a single-bottleneck configuration to study the problem. This configuration is shown in Fig. 2. The network topology of configuration (c) is the same as that of configuration (a). However, the traffic of configuration (c) is different from that of configuration (a). Now between each sending host and the receiving host, there are $N$ pairs of a web server and a greedy web client. Therefore, in total there are $7 * N$ such pairs, where $N$ is varied from 5,10 to 15 .

We did not use the CISCO AGS + router and the 3COM 3300 SM switch to do the greedy HTTP traffic experiments. This is because (1) the FreeBSD router can provide more internal information than these commercial router/switch, and (2) the greedy TCP traffic results collected on configuration (a) and (b) show that the CISCO AGS + router and the 3COM $3300 \mathrm{SM}$ switch generate the same findings as the FreeBSD router (see Section 6). Thus, in

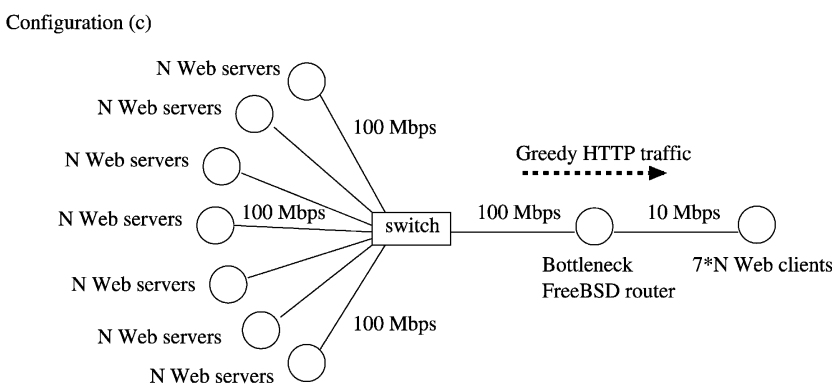

Fig. 2. The configuration used in the greedy HTTP traffic experiments 
the greedy HTTP traffic case, we just used configuration (c) to collect experimental results.

\section{Measurement method and tool}

This section presents (1) the method that we used to capture the queue length variation, packet arrival, departure, and dropping processes occurring inside a bottleneck FreeBSD router, and (2) the method that we used to calculate the packet drop rate of a bottleneck commercial router/switch.

\subsection{Capturing events}

We used the user-level tcpdump program with a modified kernel to capture the queue length variation process, packet arrival, departure, and dropping events in the FreeBSD router. The tcpdump is a user-level program that can instruct the in-kernel Berkeley packet filter [10] to capture certain packets, associate each captured packet with a timestamp (with a granularity of $1 \mu \mathrm{s}$ ), and pass the information of captured packets to the user level. Since we also wanted to pass the in-kernel events of packet arrival, departure, dropping, and the current queue length information to the user level, we decided to take advantage of tcpdump to do this job.

We modified the kernel so that the in-kernel packet filter captures only the packets that are of interest to us. By default, the packet filter captures the incoming and outgoing packets of a monitored network interface. However, this is not what we want. Actually, for a monitored interface, we are not interested in capturing its incoming packets. Instead, we want to capture three different kinds of packets. The first kind is the packets that are successfully enqueued into the output queue, the second kind is the packets that should be enqueued but dropped due to a full queue under FIFO (or selected to be dropped under RED [11]), the third kind is the packets that are dequeued from the output queue for transmission. For each of such packets, we associate it with a timestamp to record when the corresponding event (arrival, dropping, or departure) happens.

When we process the tcpdump output, we need to distinguish between these three different kinds of packets. To be able to do this, we put this 'kind' information into the Ethernet source address field of a captured packet. We did this because when a packet is captured, a copy of the packet is generated and passed to the tcpdump for display. Modifying the Ethernet header of the copy (the captured packet) to convey some information to the user level does no harm to the original packet. We set the Ethernet source address of a captured packet that is enqueued to the output queue, that is dropped, and that is dequeued from the output queue to be qlen:1:1:1:1:1, qlen:2:2:2:2:2, and qlen:3:3:3:3:3, respectively, where qlen is the current queue length when the corresponding event occurs. Using

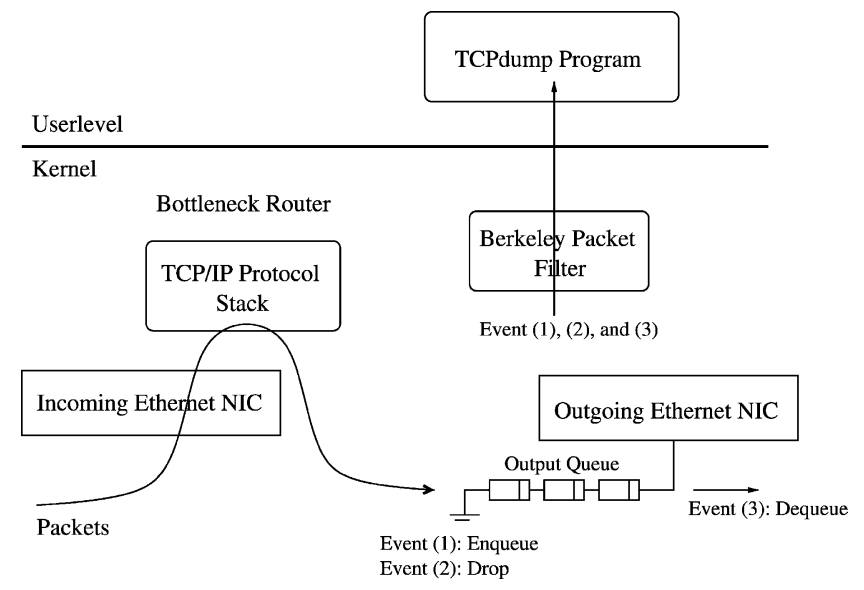

Fig. 3. The mechanism used to capture the internal queue length variation process of a bottleneck router.

this approach, the tcpdump output provides detailed information about the queue length variation, packet arrival, dropping, and departure processes. Fig. 3 depicts this capturing mechanism.

During our experiments, because we used a high performance $1.6 \mathrm{GHz} \mathrm{PC}$ as the FreeBSD router and the bottleneck link bandwidth is only $10 \mathrm{Mbps}$, the tcpdump program did not lose any packet when capturing packets.

\subsection{Calculating packet drop rates}

If a commercial router/switch does not provide packet drop count information for its output ports, we use the method presented here to calculate the packet drop rate of an output port. Not every commercial router/switch provides packet drop count information. For example, the 3COM 3300 SM switch does not provide this information.

In this method, we first sum up the number of packets successfully sent on each of the seven sending hosts. This number $S$ is the total number of packets that enter the router/switch and then are directed to the bottleneck output port. Suppose that the number of packets successfully received by the receiving host is $R$. Then the number $D$, which is $S-R$, is the number of packets dropped at the bottleneck output port. Finally, we divide $D$ by $S$ to obtain the packet drop rate of the bottleneck output port. We have verified the correctness of this method by using the FreeBSD router. In all of our verification experiments, the packet drop rate calculated by this method is very close to the real packet drop rate of the FreeBSD router.

\section{Results and findings: the greedy TCP traffic case}

In this section, we present experimental results and findings derived when the network traffic is greedy TCP traffic. As described in Section 4.1, $7 * N$ greedy TCP flows compete for the bottleneck link's bandwidth $(N=4,8$, and 12), and some packets of these TCP flows are dropped inside 
Table 1

The FIFO packet drop rate results when a FreeBSD router is used (greedy TCP traffic)

\begin{tabular}{llllllllrrrr}
\hline \# TCPs & ping1 $(\%)$ & ping2 $(\%)$ & ping3 $(\%)$ & ping4 (\%) & ping5 (\%) & ping6 (\%) & ping7 (\%) & ave (\%) & qFull & Real $(\%)$ & ave/r \\
\hline 28 TCPs & 10.66 & 12.67 & 12.02 & 11.79 & 11.80 & 11.21 & 11.97 & 11.73 & 11.30 & 3.85 & 2.93 \\
56 TCPs & 16.56 & 15.66 & 15.97 & 15.74 & 14.83 & 15.84 & 15.47 & 15.72 & 15.01 & 6.80 & 2.20 \\
84 TCPs & 19.49 & 18.43 & 19.52 & 19.09 & 19.68 & 18.38 & 19.19 & 19.11 & 18.20 & 9.18 & 1.98 \\
\hline
\end{tabular}

the bottleneck router/switch. Every experiment lasts at least $90 \mathrm{~min}$. In the following, more specific setup information will be provided.

\subsection{Packet drop rate measurements}

With the background greedy TCP traffic going on, we run the ping program on each of the seven sending hosts to measure the packet drop rate of the paths between the sending and receiving hosts. The ping program sends multiple ping request packets to the receiving host, and requires the receiving host to send back a ping reply packet when it receives a ping request packet. When the ping program stops, it divides the number of received reply packets by the number of sent request packets and reports this number as the measured packet drop rate. Because there is no bottleneck on the paths from the receiving host to the sending hosts, only ping request packets may be dropped and no ping reply packet will be dropped. The FreeBSD router, CISCO AGS + router, and 3COM 3300 SM switch do not favor non-ping packets over ping packets or favor ping packets over non-ping packets when dropping packets. Therefore, the seven ping programs unbiasedly measure the packet drop rate that a packet may experience at the bottleneck output port.

Each of these ping programs uses the default time interval of $1 \mathrm{~s}$ to send out its request packets. The number of request packets sent during every experiment is at least 5000. (We need this large number of samples to obtain converged results. See the figures and discussion in Section 6.4.) Because each request packet is only 84 bytes and each ping program's sending rate is 1 packet/s, the extra traffic load placed on the bottleneck link by the seven ping programs is only 588 bytes/s. Since this rate is only $0.045 \%$ of the bottleneck link's bandwidth (10 Mbps), the ping probe packets affect the original background TCP traffic insignificantly. Our experimental results show that the bottleneck output port's packet drop rate is almost the same with or without the ping traffic.

\subsection{Finding about packet drop rate under FIFO}

Finding 1. When FIFO is used in a bottleneck router/switch, the measured packet drop rate reflects the queue-full percentage, which is larger than the real packet drop rate.

The bottleneck routers/switches that we used to verify this finding are (1) a FreeBSD router, (2) a CISCO AGS + router, and (3) a 3COM $3300 \mathrm{SM}$ switch. In (1)-(3), the packet scheduling and dropping scheme used at the bottleneck output port is FIFO and drop tail. The maximum queue lengths of the FIFOs in (1) and (2) are the default of 50 and 40 packets, respectively. The information about the maximum queue length of the FIFO in (3) is unavailable. However, because the transmission time of a 1500-byte TCP packet on the $10 \mathrm{Mbps}$ bottleneck link is $1.2 \mathrm{~ms}$ and the maximum queueing delays reported by ping packets in (3) is $158 \mathrm{~ms}$, we estimate that the maximum queue length of the 3COM 3300 SM switch is about 130 (158/1.2) packets.

Table 1 presents the results when the bottleneck is a FreeBSD router under three different levels of load $(28,56$, and 84 greedy TCP flows, respectively). The results include the packet drop rates measured by the seven ping programs, the average of these seven ping results (ave), the queue-full percentage, the real packet drop rate (real), and the ratio of ave over real (ave/r). Table 2 presents the results when the bottleneck is a CISCO AGS + router. Table 3 presents the results when the bottleneck is a 3COM $3300 \mathrm{SM}$ switch.

From Tables 1 to 3 , we see that the packet drop rates measured by the seven ping programs overestimate the real packet drop rate (the overestimating ratios are between 1.45 and 2.93), and what they really measure is the queue-full percentage rather than the real packet drop rate. This important finding can be explained as follows.

First, when a probe packet arrives at a bottleneck output port, it must be in either a queue-full period or a non-queuefull period. If it is in a queue-full period, the probe packet must be dropped. Otherwise, it is not dropped. It is clear that the packet drop rate measured (experienced) by multiple

Table 2

The FIFO packet drop rate results when a CISCO AGS + router is used (greedy TCP traffic)

\begin{tabular}{|c|c|c|c|c|c|c|c|c|c|c|c|}
\hline \# TCPs & ping1 (\%) & ping2 (\%) & ping3 $(\%)$ & ping4 $(\%)$ & ping5 (\%) & ping6 (\%) & ping7 (\%) & ave $(\%)$ & qFull & Real (\%) & ave/r \\
\hline 28 TCPs & 13.50 & 13.21 & 13.56 & 12.87 & 12.76 & 13.23 & 12.42 & 13.07 & N/A & 4.96 & 2.63 \\
\hline 56 TCPs & 15.37 & 15.45 & 16.26 & 17.31 & 17.58 & 16.72 & 16.49 & 16.65 & N/A & 6.60 & 2.49 \\
\hline 84 TCPs & 19.37 & 18.45 & 19.83 & 19.96 & 18.69 & 19.81 & 19.83 & 19.42 & N/A & 8.47 & 2.29 \\
\hline
\end{tabular}


Table 3

The FIFO packet drop rate results when a 3COM 3300 SM switch is used (greedy TCP traffic)

\begin{tabular}{|c|c|c|c|c|c|c|c|c|c|c|c|}
\hline \# TCPs & ping1 (\%) & ping2 (\%) & ping3 $(\%)$ & ping4 $(\%)$ & ping5 (\%) & ping6 $(\%)$ & ping7 (\%) & ave $(\%)$ & qFull & Real (\%) & ave/r \\
\hline $28 \mathrm{TCPs}$ & 4.36 & 4.49 & 4.49 & 4.02 & 4.05 & 4.27 & 4.70 & 4.34 & N/A & 2.36 & 1.83 \\
\hline 56 TCPs & 8.24 & 8.24 & 8.22 & 8.39 & 8.25 & 8.34 & 8.56 & 8.32 & N/A & 5.45 & 1.52 \\
\hline 84 TCPs & 10.64 & 11.25 & 12.03 & 11.97 & 12.23 & 11.71 & 11.69 & 11.64 & N/A & 7.99 & 1.45 \\
\hline
\end{tabular}

probe packets in the time domain actually reflects the queuefull percentage.

Second, because in a queue-full period there may be no packet dropping (to be discussed later) whereas a probe packet arriving in such a period must be dropped, the queuefull percentage does not proportionally correspond to the real packet drop rate.

In a queue-full period, there may be no packet dropping. Such a period is called 'queue-full-but-no-drop' in this paper and may occur as follows. Suppose that the current queue length is maxqlen-1. When a packet arrives and is enqueued to the output queue, the current queue length will go up to maxqlen, which starts a queue-full period. Later, when an in-queue packet is dequeued for transmission, the current queue length will go down to maxqlen-1, which stops the current queue-full period. If no other packet arrives in this period, no packet is dropped in this queue-full period.

The queue-full percentage is greater than the real packet drop rate in experiments. This is because in these experiments, the average packet drop rate (measured in packet/s) in queue-full periods is less than the average packet arrival rate. This phenomenon certainly must occur in these experiments. Otherwise, the real packet drop rate will be equal to or greater than the queue-full percentage, which is not true for these experiments. (Note: For an experiment, let $N$ and $D$ represent the number of packet arrival and dropping events, and $F$ and $T$ represent the total time of queue-full periods and the total time of the experiment. If $D / F<N / T$ is not true, then $D / F \geq N / T$ must be true. This is equivalent to $D / N \geq F / T$, which means that the real packet drop rate is equal to or greater than the queue-full percentage and is not true.) We use the experimental results of the 28 TCPs case of Table 1 to show this phenomenon. In the experiment, the average packet drop rate in queue-full periods is 286.21 packet/s. This number is less than the average packet arrival rate of $840.61 \mathrm{packet} / \mathrm{s}$. The results of other cases also exhibit the same phenomenon.

When network congestion level increases, the overestimating ratio decreases. This phenomenon is evidenced by the results in Tables $1-3$. The reason is that as network load increases, the queue-full-but-no-drop periods occur less and less likely. This causes the ratio (denoted as $P D R Q F / P A R)$ of the average packet drop rate in queue-full periods (measured in packet/s) over the average packet arrival rate to increase. As an evidence, for the 28 TCPs, 56 TCPs, and 84 TCPs cases of Table 1 , the ratios of queuefull-but-no-drop percentage over queue-full percentage are
$0.69,0.60$, and 0.54 , respectively (decreasing), and the ratios of $P D R Q F / P A R$ are $0.34,0.45$, and 0.51 , respectively (increasing). This finding leads to Finding 3 (presented later), which discusses how the overestimating ratio will change under FIFO and RED when network load increases.

We can derive some implications from this finding.

- If routers/switches of a network use the FIFO scheme, an end-to-end probe method (e.g. the most commonly used ping) will measure the real packet drop rate too high.

Since most network users do not carefully distinguish between a measured and the real packet drop rate of a network, they usually incorrectly interpret a measured packet drop rate as the real packet drop rate. Therefore, it is unfair to their ISPs because the quality of their networks actually is not that bad.

- A network's packet drop rate is a composite number. Different types of traffic in a network may experience and have different packet drop rates. This implication is evidenced by the different packet drop rates experienced by probe packets and the background TCP packets listed in Tables 1-3. If we view the seven ping packet streams as a part of the background traffic in the first beginning and do not view them as external probe packets, clearly we see that TCP and ping packets experience and have different packet drop rates at the same bottleneck.

Why ping and TCP packets experience different packet drop rates can be explained as follows. Because ping packets are transmitted independently of the packet drop rate experienced by previously sent ping packets, they unbiasedly sample the length of the bottleneck output queue. The ratio of the number of ping packets that encounter a queue-full period over the total number of ping packets sent thus will converge to the queue-full percentage. TCP packets, on the other hand, are sent under the control of TCP congestion control, whose actions depend on the packet drop rate experienced by previously sent TCP packets. Because TCP packets are not independently sent, they do not unbiasedly sample the length of the bottleneck output queue. Therefore, the packet-dropping ratio (measured packet drop rate) does not correspond to the queue-full percentage.

- If the majority of traffic is TCP, using the packets of a greedy TCP flow as probe packets can obtain a closer estimate of the real packet drop rate.

In our experiments, the traffic flows that compete for the bottleneck link's bandwidth are all TCP flows and they are homogeneous. Each of these TCP flows thus will 
Table 4

The RED packet drop rate results when a FreeBSD router is used (greedy TCP traffic)

\begin{tabular}{|c|c|c|c|c|c|c|c|c|c|c|c|}
\hline \# TCPs & ping1 (\%) & ping2 (\%) & ping3 (\%) & ping4 $(\%)$ & ping5 (\%) & ping6 (\%) & ping7 (\%) & ave $(\%)$ & forceD $(\%)$ & Real (\%) & ave/r \\
\hline 28 TCPs & 4.78 & 4.96 & 4.82 & 5.08 & 4.99 & 4.78 & 4.87 & 4.89 & 0.08 & 4.68 & 1.04 \\
\hline 56 TCPs & 7.21 & 7.31 & 7.85 & 8.33 & 8.68 & 8.25 & 9.01 & 8.09 & 0.6 & 7.05 & 1.14 \\
\hline 84 TCPs & 10.58 & 10.84 & 10.73 & 11.78 & 11.29 & 11.64 & 12.22 & 11.29 & 1.91 & 9.03 & 1.25 \\
\hline
\end{tabular}

experience the same packet drop rate (the real packet drop rate of the network). This means that if we use the packets of a greedy TCP flow as probe packets, the packet drop rate measured by them will reflect the real packet drop rate. This implication is confirmed by a post analysis of our experimental results. For example, for the 28 TCP flows case of Table 1, the packet drop rates experienced by five individual TCP flows are 3.74, 3.80, $3.81,3.76,3.98$, respectively. These numbers are close to the real packet drop rate.

\subsection{Finding about packet drop rate under RED}

Finding 2. When RED is used in a bottleneck router and the network is not too congested, the measured packet drop rates are close to the real packet drop.

The bottleneck router that we used to verify this finding is a FreeBSD router. Unlike what we did for the FIFO case, because the CISCO AGS + router and 3COM 3300 SM switch do not support RED, they are not used in the experiments. To use RED at the bottleneck output port of the FreeBSD router, we let the router run FreeBSD 4.2 and the ALTQ 3.0 [12] package. The ALTQ package implements various packet scheduling and buffer management schemes for a PC-UNIX based router. The parameter settings for RED used in our experiments are (weight:1/512 inv_pmax:10 qthresh:(10,50) q_limit:50).

Table 4 presents the results when the bottleneck router uses RED under three different levels of load (28,56, and 84 greedy TCP flows, respectively). The information shown in this table is similar to that shown in previous tables. The only exception is that the qFull column is replaced with the forceD (forced drop) column, which shows what percentage of incoming packets is force-dropped. The design of RED tries to drop incoming packets proactively before the queue length exceeds the maximum queue length. The queue-full percentage under RED thus is very small when the network is not too congested. However, if the network becomes severely congested and the queue eventually overflows, some packets need to be dropped (called 'forced drop' in RED).

From Table 4, we see that for the 28 TCP flows case, the packet drop rates measured by the seven ping programs are very close to the real packet drop rate. (The overestimating ratio is only 1.04). For the 56, and 84 TCP flows cases, although the overestimating ratio becomes larger and increases to 1.14 and 1.25 , respectively, the measured and real packet drop rates are still quite close to each other, compared to the FIFO results.

REDs proactive packet dropping strategy makes a measured packet drop rate close to the real packet drop rate. In RED, when a packet arrives at an output port, the probability of dropping it is a linear function $\operatorname{Drop}($ ) of the exponential average of the queue length. Suppose that the probability distribution of the exponential average queue length seen by arriving packets is $P(i), i=0,1,2, \ldots$, maxqlen, the average packet dropping probability (or drop rate) for arriving packets will be $\sum_{i=0}^{\operatorname{maxqlen}} P(i) * \operatorname{Drop}(i)$.

The probability distribution of the exponential average queue length seen by probe packets is close to that seen by TCP packets under RED. Fig. 4 shows the probability distributions of the 28 TCP flows case of Table 4. (Note: Because probe packets are ICMP packets, we can distinguish probe packets from TCP packets in a tcpdump $\log$ file.) Because the difference between $P(i)_{\text {probe }}$ (seen by probe packets) and $P(i)_{\mathrm{TCP}}$ (seen by TCP packets) is small and less than 1 (the maximum difference is 0.003 in Fig. 4 when $i$ is 32), and $\operatorname{Drop}(i)$ is also small and less than 1 (the maximum value is 0.1 when $i$ is maxqlen), the product term $\left(P(i)_{\text {probe }}-P(i)_{\mathrm{TCP}}\right) * \operatorname{Drop}(i)$ becomes tiny (the maximum value is 0.000165 when $i$ is 32). Because every product term is tiny and some of the product terms are positive while some of them are negative, the sum of these product terms, which is the difference between the packet drop rates of probe and TCP packets, is small (0.0015).

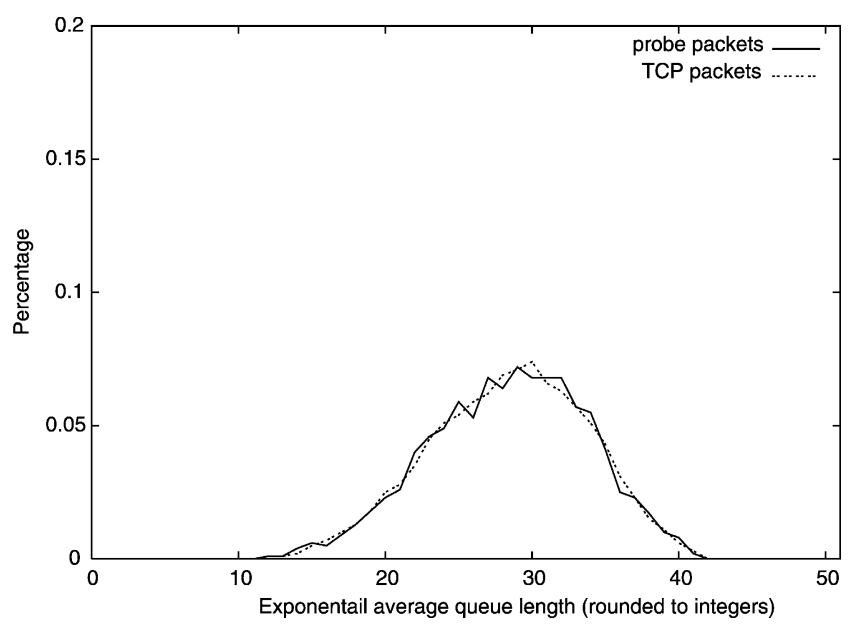

Fig. 4. The probability distributions of the exponential average queue length (rounded to integers) seen by probe and TCP packets under RED, respectively (greedy TCP traffic). 
Using the same $\sum_{i=0}^{\max q \text { len }} P(i) * \operatorname{Drop}(i)$ formula, we can show that under FIFO the packet drop rate difference is not tiny. Under FIFO, because Drop(maxqlen) is 1 and $\operatorname{Drop}(i)$ is $0, i=0,1,2, \ldots$, maxqlen- 1 , the packet drop rate difference becomes $\left(P(\text { maxqlen })_{\text {probe }}-P(\text { maxqlen })_{\mathrm{TCP}}\right) * 1$. $P(\text { maxqlen })_{\text {probe }}-P(\text { maxqlen })_{\mathrm{TCP}}$ is close to the queue-fullbut-no-drop percentage and is not tiny. Fig. 5 shows the probability distributions of the 28 TCP flows case of Table 1 . The difference between $P(\text { maxqlen })_{\text {probe }}$ and $P(\text { maxqlen })_{\mathrm{TCP}}$ is about $7 \%$ (maxqlen here is 50 ), which is not tiny.

Finding 3. When network load increases, the overestimating ratio under RED increases whereas the overestimating ratio under FIFO decreases.

When the network congestion level increases and causes a higher percentage of incoming packets to be forcedropped, the overestimating ratio under RED will increase. Table 4 shows that when the load increases from 28,56 , to 84 greedy TCP flows, the forced drop percentage increases from $0.08,0.6$, to $1.91 \%$, and the overestimating ratio increases from $1.04,1.14$, to 1.25 . This can be explained as follows. When a larger and larger percentage of packets are force-dropped, RED gradually degenerates to the FIFO scheme. Because our previous FIFO results show that the FIFO scheme has the overestimation problem, it is clear that when network load becomes heavier, a measured packet drop rate will deviate from the real packet drop rate by a larger value.

Previously, our experimental results show that when network load increases, the overestimating ratio under FIFO decreases. It is interesting to calculate the packet drop rate where the two ratios will meet each other. (Note: Because even under severe congestion, RED at worst only degenerates to FIFO and will not perform worse than FIFO, these two ratios will not cross over.) Using the regression analysis and extrapolation method, we find that this rate would be $13 \%$ for the experiments of Tables 1 and 4 .

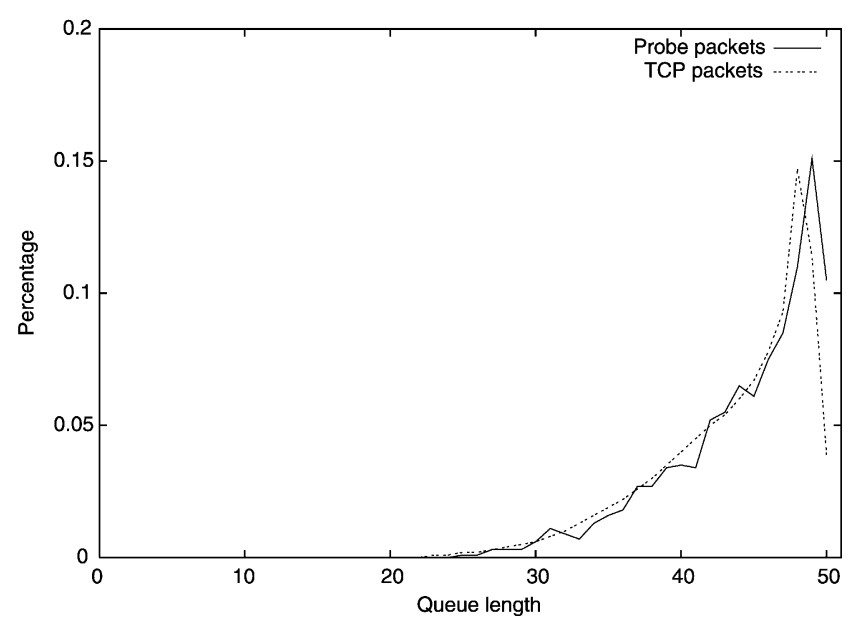

Fig. 5. The probability distributions of the queue length seen by probe and TCP packets under FIFO, respectively (greedy TCP traffic).

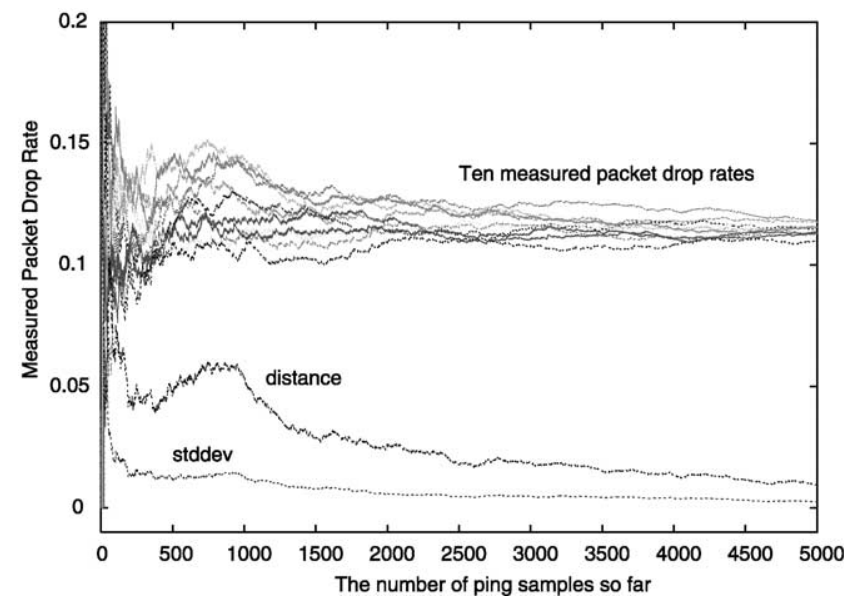

Fig. 6. The convergence of measured packet drop rates under FIFO when periodic sampling is used (greedy TCP traffic).

\subsection{Finding about various sampling techniques}

Finding 4. Different sampling techniques generate close measured packet drop rates and convergence speeds.

When using probe packets to measure a network's packet drop rate, an important question is "Will different distributions of inter-probe packet times result in different measured packet drop rates and convergence speeds?" By 'convergence speed', we mean how many samples are needed to let a measured packet drop rate converge to what it should eventually converge to. Another important question is "If we have multiple probe packet streams that measure the same bottleneck router's packet drop rate but each of them is launched at a different time, how soon can their results approach each other?" (Note: In this case, we are concerned about the convergence of the difference among their results, not the convergence of the difference between their results and the real packet drop rate.)

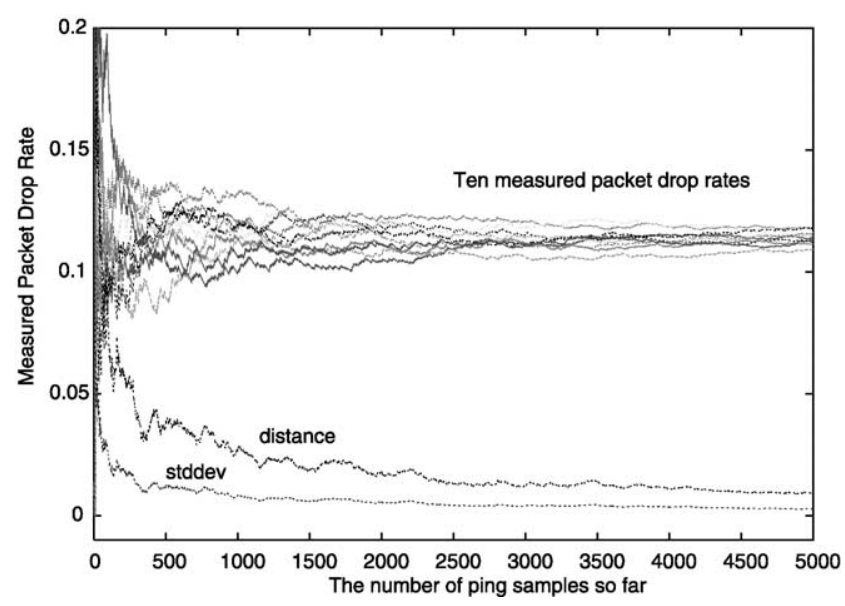

Fig. 7. The convergence of measured packet drop rates under FIFO when periodic sampling with random delay is used (greedy TCP traffic). 


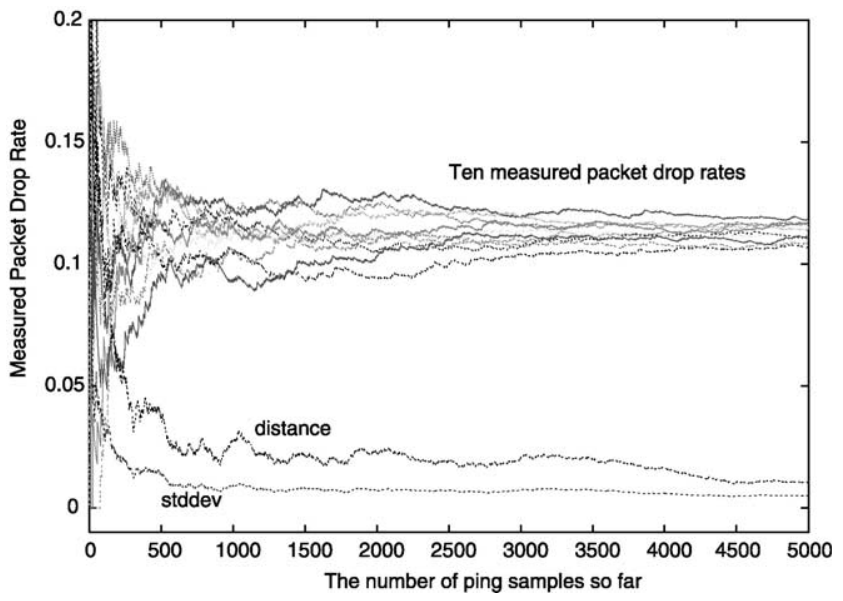

Fig. 8. The convergence of measured packet drop rates under FIFO when Poisson sampling is used (greedy TCP traffic).

To answer these questions, we used two queue length variation processes collected in the FreeBSD router to perform some post analyses. The first was collected when the FreeBSD router uses the FIFO scheme and under the load of 28 greedy TCP flows (the first row of Table 1). The second was collected with RED and under the same load (the first row of Table 4).

We test three different sampling techniques (periodic, periodic + random delay, Poisson) in our analyses. The periodic sampling is the most widely used method for its simplicity. In this technique, the inter-arrival times of probe packets at the bottleneck output port is a fixed interval. The periodic + random delay sampling is used to simulate the delay jitter that periodic probe packets may experience before arriving at the output port. In analyses, we add a random number generated uniformly between -30 and $30 \mathrm{~ms}$ to a packet's original arrival time. That is, if the average interval is $T$ seconds, the arrival time of the $i$ th probe packet is set to be $T * i+r_{i}$, where $r_{i}$ is a uniform random number between -30 and $30 \mathrm{~ms}$. We also study Poisson

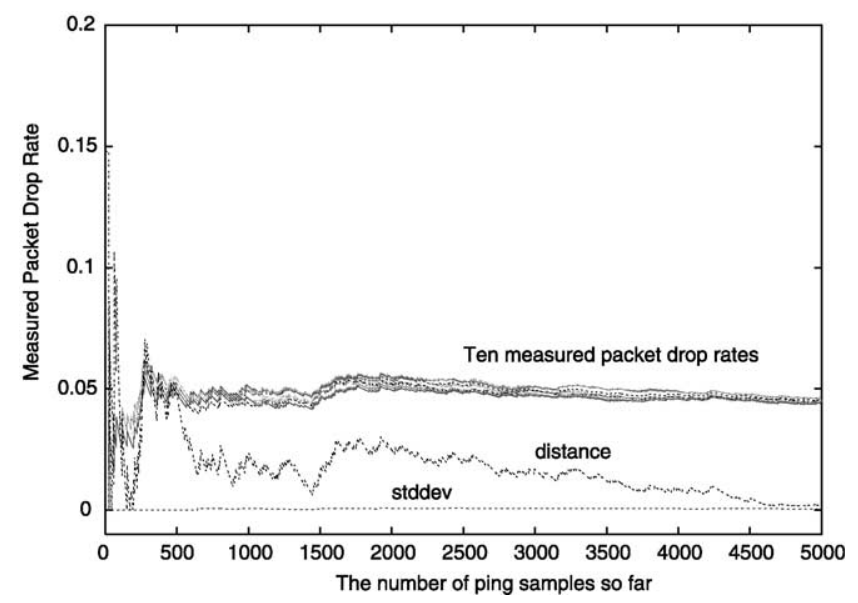

Fig. 9. The convergence of measured packet drop rates under RED when periodic sampling is used (greedy TCP traffic).

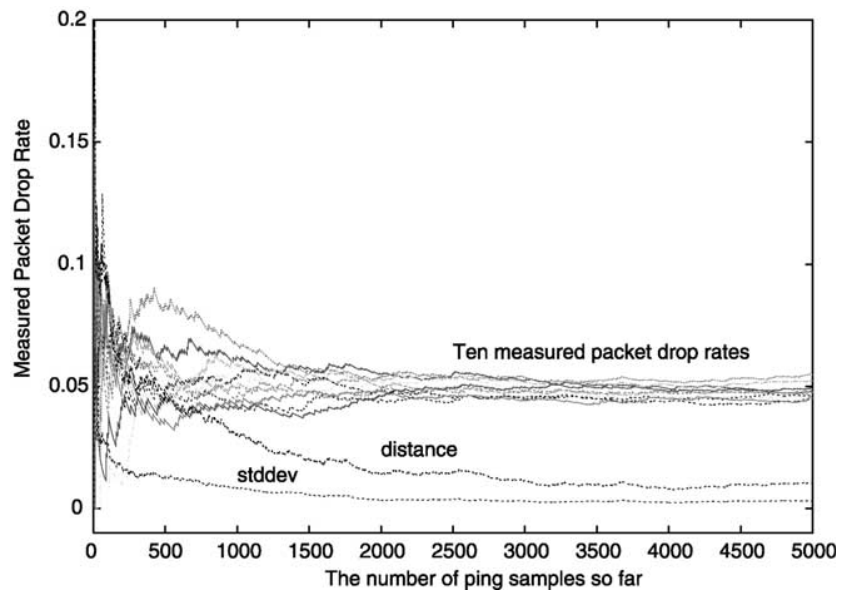

Fig. 10. The convergence of measured packet drop rates under RED when periodic sampling with random delay is used (greedy TCP traffic).

sampling because it is recommended in Ref. [13] for its memoryless property [14]. In our analyses, the average interarrival time for these sampling techniques is set to the same value of $1 \mathrm{~s}$ (the default value used by the ping program).

In our analyses, for a $N$-packet probe packet stream, given the arrival times of its packets $P_{1}, P_{2}, \ldots, P_{N}$, the measured packet drop rate up to the time when packet $P_{i}$ is issued is calculated as $D / i$, where $D$ is the number of packets between $P_{1}$ and $P_{i}$ that are dropped due to encountering a full queue under FIFO or are selected to be dropped under RED. Under FIFO, we consider a packet to be dropped if its arrival time fall into a queue-full period of the collected queue length variation process. Under RED, we consider a packet to be dropped if the dropping probability function chooses to drop it. To study the convergence speeds of a sampling technique, in each analysis, we launch ten probe packet streams using the same sampling technique at $0.0,0.1,0.2, \ldots, 0.9 \mathrm{~s}$, respectively. If a probe packet stream needs to use random numbers (e.g. Poisson sampling), it uses a different sequence of random numbers.

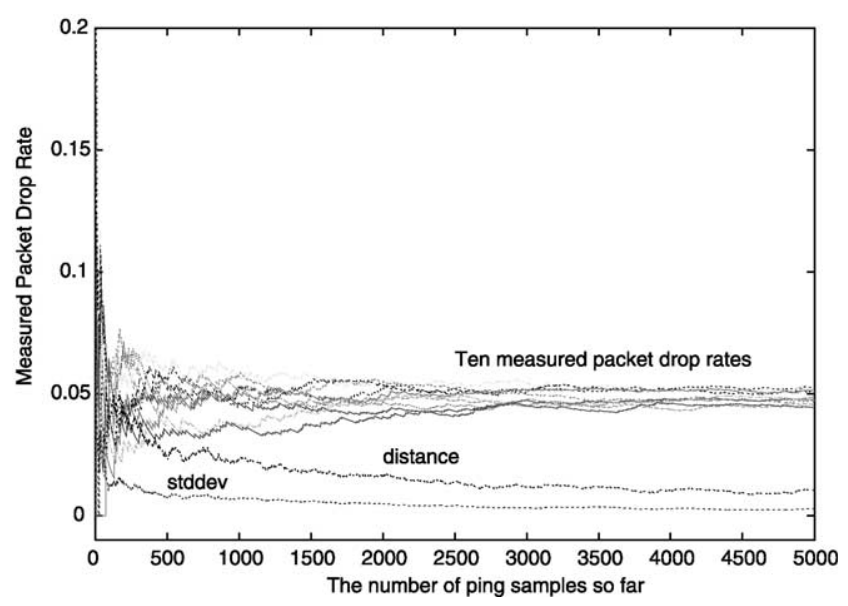

Fig. 11. The convergence of measured packet drop rates under RED when Poisson sampling is used (greedy TCP traffic). 
Table 5

The FIFO packet drop rate results when a FreeBSD router is used (greedy HTTP traffic)

\begin{tabular}{lrrrrrrrrrrr}
\hline \# HTTPs & ping1 $(\%)$ & ping2 $(\%)$ & ping3 $(\%)$ & ping4 (\%) & ping5 (\%) & ping6 (\%) & ping7 (\%) & ave (\%) & qFull (\%) & Real (\%) & ave/r \\
\hline 35 HTTPs & 3.23 & 3.05 & 3.12 & 2.98 & 2.95 & 3.20 & 3.03 & 3.08 & 3.01 & 2.16 & 1.43 \\
70 HTTPs & 8.19 & 8.17 & 7.88 & 7.94 & 7.99 & 8.06 & 8.28 & 8.07 & 7.91 & 6.17 & 1.31 \\
105 HTTPs & 10.49 & 11.17 & 10.74 & 10.53 & 11.43 & 10.87 & 10.89 & 10.92 & 10.63 & 9.34 & 1.17 \\
\hline
\end{tabular}

Figs. $6-8$ present the packet drop rates measured by 10 probe packet streams under FIFO. Figs. 9-11 present the 10 measured results under RED. In these figures, each of the 10 lines in the top represents the measured packet drop rate of a probe packet stream. The line at the bottom represents the standard deviation (labeled 'stddev') of these 10 measured packet drop rates. This metric shows how soon these measured drop rates approach each other. The line above the stddev line represents the distance (labeled 'distance') between the 10 measured packet drop rates and the packet drop rate $C$ that they should converge to. (Note: We have shown that under FIFO, this value is the queue-full percentage, and under RED, it is the real packet drop rate.) More precisely, suppose that $M_{1}, M_{2}, \ldots, M_{10}$ are the 10 measured packet drop rates, then the distance is the distance between the two points $(C, C, C, C, C, C, C, C, C, C)$ and $\left(M_{1}, M_{2}, M_{3}, M_{4}, M_{5}, M_{6}, M_{7}, M_{8}, M_{9}, M_{10}\right)$ in the 10-dimension space $\left(\sqrt{\sum_{i=1}^{10}\left(M_{i}-C\right)^{2}}\right)$. This metric shows how soon the measured packet drop rates converge to what they should eventually converge to.

From these figures, we have some findings as follow.

- Different sampling techniques generate close measured packet drop rates.

In the FIFO case, the periodic, periodic + random delay, and Poisson samplings all converge to the queuefull percentage. In the RED case, they all converge to the real packet drop rate. In fact, we have tried many different distributions of the inter-arrival times of probe packets (e.g. the normal and uniform distributions). They all generate close packet drop rates.

- Different sampling techniques have about the same convergence speeds.

In both the FIFO and RED cases, from the stddev and distance lines, we see that the three different sampling techniques have about the same convergence speeds.

- Under RED, different probe packet streams report very close measured packet drop rates over time if their corresponding packets (i.e. $P_{i}$ ) arrive at the bottleneck output port not too far away from each other.

RED uses the exponential average of the queue length to calculate a dropping probability. Its weighing factor is 1/512 for a new queue length sample. (Note: The exponential average of the queue length is updated as $\mathrm{EA}=\mathrm{EA} * 511 / 512+$ NewQlen $* 1 / 512$, where $\mathrm{EA}$ is the exponential average and NewQlen is a new sample of the current queue length.) Because the weighting factor is purposely configured small to not punish bursty traffic, the exponential average changes very slowly. Therefore, if probe packets arrive closely at the output port, they would receive about the same packet dropping probabilities.

This explains why in Fig. 9, the standard deviation of the 10 measured packet drop rates reduces to a small value rapidly while the distance to the real packet drop rate is still large. In Fig 9, the 10 periodic sampling packet streams are only shifted by $0.1 \mathrm{~s}$ on the time axis and use no random number in their scheduled arrival times. Therefore, the arrival times of packet $P_{i}$ of each of the 10 probe packet streams are close to each other.

- A large number of samples are needed to obtain converged results.

We see that for a probe packet stream to obtain a measured packet drop rate that is close to what it should converge to, just a few hundreds of probe packets are not enough. At least a few thousands probe packets are needed to let a measured packet drop rate approach what it should converge to.

\section{Results and findings: the greedy HTTP traffic case}

In this section, we present experimental results and findings derived when the network traffic is greedy HTTP traffic. As described in Section 4.2, the reply traffic of $7 * N$

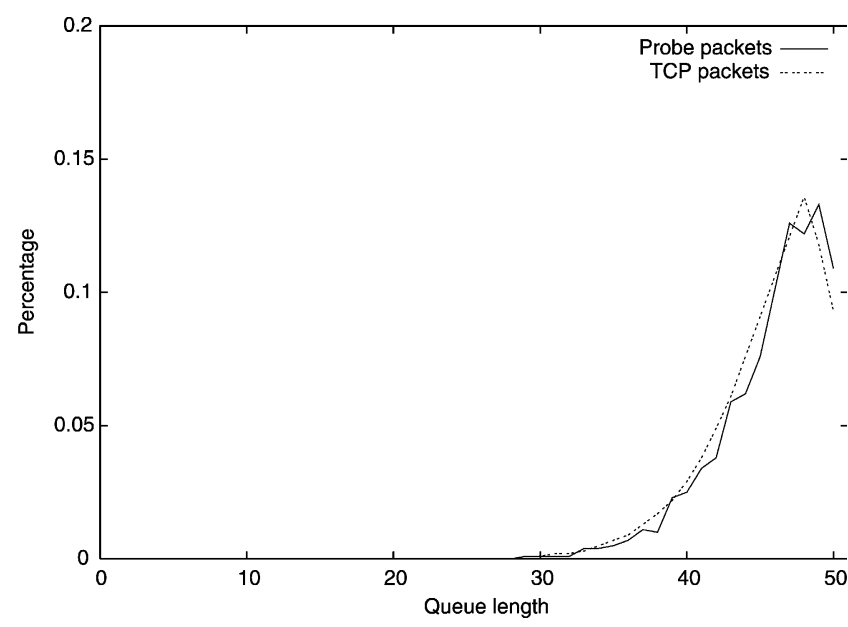

Fig. 12. The probability distributions of the queue length seen by probe and TCP packets under FIFO, respectively (greedy HTTP traffic). 
Table 6

The RED packet drop rate results when a FreeBSD router is used (greedy HTTP traffic)

\begin{tabular}{|c|c|c|c|c|c|c|c|c|c|c|c|}
\hline \# HTTPs & ping1 (\%) & ping2 $(\%)$ & ping3 (\%) & ping4 (\%) & ping5 (\%) & ping6 (\%) & ping7 (\%) & ave $(\%)$ & forceD $(\%)$ & Real (\%) & ave/r \\
\hline 35 HTTPs & 3.23 & 3.01 & 3.10 & 3.20 & 3.07 & 3.38 & 3.27 & 3.18 & 0.007 & 3.14 & 1.01 \\
\hline 70 HTTPs & 5.69 & 5.74 & 6.00 & 5.77 & 5.84 & 5.92 & 5.79 & 5.82 & 0.076 & 5.65 & 1.03 \\
\hline 105 HTTPs & 7.63 & 7.54 & 7.42 & 7.64 & 7.37 & 7.54 & 7.49 & 7.51 & 0.870 & 7.08 & 1.06 \\
\hline
\end{tabular}

greedy Web clients compete for the bottleneck link's bandwidth $(N=5,10$, and 15). The size of each web transfer is $16 \mathrm{~KB}$. Every experiment lasts at least $90 \mathrm{~min}$.

\subsection{Packet drop rate measurements}

Like what we did in the greedy TCP traffic case, with greedy HTTP traffic going on, we run the ping program on each of the seven sending hosts to measure the packet drop rate of the bottleneck FreeBSD router. The interval used by the ping program for its request packets is the default value of $1 \mathrm{~s}$.

\subsection{Finding about packet drop rate under FIFO}

Table 5 presents the FIFO results under three different levels of load (35, 70, and 105 greedy HTTP sessions, respectively). Like the results presented in Table 1 , the results in Table 5 show that the measured packet drop rates are higher than the real packet drop rates of the bottleneck router and actually are close to the queue-full percentages. These results show that Finding 1 , which is presented in Section 6.2, also holds for the greedy HTTP traffic case.

Fig. 12 shows the probability distributions of the queue length seen by probe packets and TCP packets under FIFO for the 105 HTTP sessions case of Table 6. We can see that probe packets experience more packet drops than TCP packets (at the place when the queue length is 50), and their

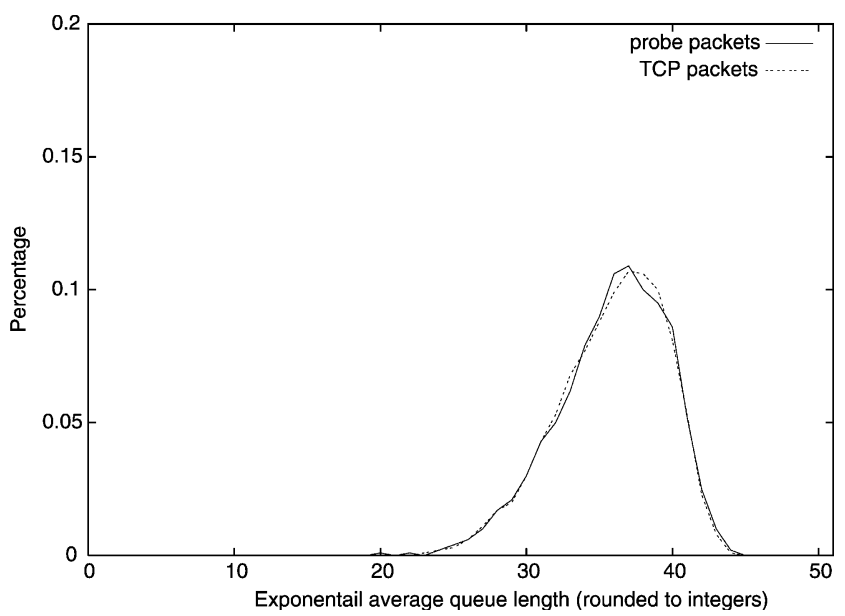

Fig. 13. The probability distributions of the exponential average queue length (rounded to integers) seen by probe and TCP packets under RED, respectively (greedy HTTP traffic). experienced packet drop rate is close to the queue-full percentage.

\subsection{Finding about packet drop rate under RED}

Table 6 presents the RED results under three different levels of load (35, 70, and 105 greedy HTTP sessions, respectively). Like the results presented in Table 4, the results in Table 6 show that the measured packet drop rates are close to the real packet drop rates of the bottleneck router. These results show that Finding 2, which is presented in Section 6.3, also holds for the greedy HTTP traffic case.

By comparing the results shown in Tables 5 and 6 , we can find that Finding 3, which is presented in Section 6.3, holds for the greedy HTTP traffic case as well. That is, under FIFO, the overestimating ratio decreases as the network load increases whereas under RED, the overestimating ratio increases as the network load increases.

Fig. 13 shows the probability distributions of the exponential average queue length seen by probe packets and TCP packets under RED for the 105 HTTP sessions case of Table 6. We can see that these two probability distribution curves are close to each other.

\subsection{Finding about various sampling techniques}

Like what we did in Section 6.4, we test the performance of various sampling techniques when the network traffic is greedy HTTP traffic. The performance results show that Finding 4, which is presented in Section 6.4, holds for the greedy HTTP traffic case as well. To space, we do not present the performance figures of these cases in this paper.

\section{Conclusions}

In this paper, we study several important questions related to using probe packets to measure a network's real packet drop rate. By using an instrumented FreeBSD kernel as a bottleneck router, we were able to collect its internal queue length variation process and derive the following findings.

- Finding 1. When FIFO is used in a bottleneck router/switch, the measured packet drop rate reflects the queue-full percentage, which is larger than the real packet drop rate. 
- Finding 2. When RED is used in a bottleneck router/ switch and the network is not too congested, the measured packet drop rates are close to the real packet drop rate.

- Finding 3. When network load increases, the overestimating ratio under RED increases whereas the overestimating ratio under FIFO decreases.

- Finding 4. Different sampling techniques generate close measured packet drop rates and convergence speeds.

The above findings are derived from the results collected on our single-bottleneck testbed network. In the future, we plan to build a multibottleneck testbed network and repeat our studies.

\section{Acknowledgements}

We would like to thank the anonymous reviewers for their valuable comments. This research was supported in part by MOE Program of Excellence Research under contract 89-E-FA04-4, the Lee and MTI Center for Networking Research, NCTU, NSC grant 90-2213-E-009157, and the Institute of Applied Science and Engineering Research, Academia Sinica, Taiwan.

\section{References}

[1] J.-C. Bolot, End-to-End Packet Delay and Loss Behavior in the Internet, Proceedings of SIGCOMM'93, September, 1993, pp. 289298.
[2] V. Paxson, End-to-end internet packet dynamics, IEEE/ACM Transactions on Networking 7 (3) (1999) 277-292. June.

[3] M. Yajnik, S. Moon, J. Kurose, D. Towsley, Measurement and Modeling of the Temporal Dependence in Packet Loss, Proceedings of IEEE INFOCOM'99, March, 1999.

[4] Y. Zhang, V. Paxson, S. Shenker, On the constancy of Internet path properties, Proceedings of ACM SIGCOMM Internet Measurement Workshop (IMW'2001), San Francisco, California, USA, November, 2001.

[5] R. Caceres, N.G. Duffield, J. Horowitz, D. Towsley, T. Bu, MulticastBased Inference of Network-Internal Characteristics: Accuracy of Packet Loss Estimation, Proceedings of IEEE INFOCOM'99, March, 1999.

[6] I. Cidon, A. Khamisy, M. Sidi, Analysis of packet loss processes in high-speed networks, IEEE Transactions on Information Theory 39 (1) (1993) 98-108. January.

[7] K. Claffy, G. Polyzos, H.-W. Braun, Application of Sampling Methodologies to Network Traffic Characterization, Proceedings of ACM SIGCOMM'93, September, 1993, pp. 194-203.

[8] M.S. Borella, Measurement and interpretation of internet packet loss, Journal of Communications and Networks 2 (2) (2000) June.

[9] FreeBSD General Commands Manual.

[10] S. McCanne, V. Jacobson, Berkeley Packet Filter: A New Architecture for User-Level Packet Capture, Proceedings of USENIX Winter Technical Conference, January, 1993.

[11] S. Floyd, V. Jacobson, Random early detection gateways for congestion avoidance, IEEE/ACM Transactions of Networking 1 (4) (1993) 397-413. August.

[12] K. Cho, A Framework for Alternate Queueing: Towards Traffic Management by PC-UNIX Based Routers, Proceedings of 1998 USENIX Annual Technical Conference, June, 1998, The latest ALTQ release can be retrieved from http://www.csl.sony.co.jp/kjc/software. html..

[13] V. Paxson, G. Almes, J. Mahdavi, M. Mathis, Framework for IP performance metrics, RFC 2330

[14] R. Wolf, Poisson arrivals see time averages, Operating Research 30 (2) (1982) 223-231. 